\title{
Epidemiology, Incidence and Mortality of Bladder Cancer and their Relationship with the Development Index in the World
}

\author{
Neda Mahdavifar ${ }^{1}$, Mahshid Ghoncheh $^{2}$, Reza Pakzad $^{3}, Z_{\text {Zohre Momenimovahed }}^{4}$, \\ Hamid Salehiniya ${ }^{3,5 *}$
}

\begin{abstract}
Background: Bladder cancer is an international public health problem. It is the ninth most common cancer and the fourteenth leading cause of death due to cancer worldwide. Given aging populations, the incidence of this cancer is rising. Information on the incidence and mortality of the disease, and their relationship with level of economic development is essential for better planning. The aim of the study was to investigate bladder cancer incidence and mortality rates, and their relationship with the the Human Development Index (HDI) in the world. Materials and Methods: Data were obtained from incidence and mortality rates presented by GLOBOCAN in 2012. Data on HDI and its components were extracted from the global bank site. The number and standardized incidence and mortality rates were reported by regions and the distribution of the disease were drawn in the world. For data analysis, the relationship between incidence and death rates, and HDI and its components was measured using correlation coefficients and SPSS software. The level of significance was set at 0.05 . Results: In $2012,429,793$ bladder cancer cases and 165,084 bladder death cases occurred in the world. Five countries that had the highest age-standardized incidence were Belgium 17.5 per 100,000, Lebanon 16.6/100,000, Malta 15.8/100,000, Turkey 15.2/100,000, and Denmark 14.4/100,000. Five countries that had the highest age-standardized death rates were Turkey 6.6 per 100,000, Egypt 6.5/100,000, Iraq 6.3/100,000, Lebanon 6.3/100,000, and Mali 5.2/100,000. There was a positive linear relationship between the standardized incidence rate and HDI $(r=0.653, P<0.001)$, so that there was a positive correlation between the standardized incidence rate with life expectancy at birth, average years of schooling, and the level of income per person of population. A positive linear relationship was also noted between the standardized mortality rate and $\mathrm{HDI}(\mathrm{r}=0.308, \mathrm{P}<0.001)$. There was a positive correlation between the standardized mortality rate with life expectancy at birth, average years of schooling, and the level of income per person of population. Conclusions: The incidence of bladder cancer in developed countries and parts of Africa was higher, while the highest mortality rate was observed in the countries of North Africa and the Middle East. The program for better treatment in developing countries to reduce mortality from the cancer and more detaiuled studies on the etiology of are essential.
\end{abstract}

Keywords: Incidence - mortality - bladder cancer - development index - world

Asian Pac J Cancer Prev, 17 (1), 381-386

\section{Introduction}

Cancer is a major burden of disease and public health concern in world (Keyghobadi et al., 2015; Razi et al., 2015) among cancers, Bladder cancer is an international public health problem (Malats and Real, 2015). According to the impact of the cancer on mortality, quality of life of patients suffering from the cancer and their families, and economic costs are important and remarkable issues(Klotz and Brausi, 2015). It is the ninth common cancer, the eleventh diagnosed cancer, and the fourteenth leading cause of deaths due to cancer worldwide (Ferlay et al., 2008). The cancer is considered one of the most common malignant cancers in the world, so that about 20 percent of people diagnosed eventually die because of the disease(Colombel et al., 2008; Brausi et al., 2011; Klotz and Brausi, 2015). Around 900,000 new cases are affected by the disease worldwide, 250,000 deaths occur per year due to the cancer(Klotz and Brausi, 2015) . Bladder cancer, on average, is 3 to 4 times more common in men than women. The incidence and prevalence of the cancer are seen in the sixth decade of life, especially its peak in the seventh and eighth. So is it mainly disease of elderly (Malats and Real, 2015).

The highest incidence of bladder cancer in men and women was observed in Europe, the United States, and

${ }^{1}$ Department of Epidemiology and Biostatistics, Zahedan University of Medical Sciences, Zahedan, ${ }^{2}$ Department of Epidemiology and Biostatistics, Hamadan University of medical sciences, Hamadan, ${ }^{3}$ Department of Epidemiology and Biostatistics, school of public health, Tehran University of medical sciences, Tehran, ${ }^{4}$ Qom University of Medical Sciences, Qom, ${ }^{5}$ Minimally Invasive Surgery Research Center, Iran University of Medical Sciences, Tehran, Iran*For correspondence: alesaleh70@yahoo.com 
Egypt, respectively. There was the lowest level in SubSaharan Africa, Asia, and South America, respectively. The greatest mortality rates were in parts of Europe and North Africa, and the lowest in Asia, Central America, and Middle Africa. However, the incidence and mortality have decreased in many Western countries (North America, and West and Northern Europe), but the rates have increased in some other European countries (South, Central, and East Europe) and developing countries in Asia. This pattern is due to differences in risk factors for bladder cancer (tobacco epidemic, changes in coding practices, the prevalence of Schistosoma Haematobium, especially in Africa, and occupational exposures) (Chavan et al., 2014).

The differences in risk factors are the most important reason for the difference in the incidence of and deaths from bladder cancer in the world. Risk factors for bladder cancer include smoking (responsible for $50 \%$ of bladder cancer and $65 \%$ of invasive bladder cancer (Klotz and Brausi, 2015)), infection with Schistosoma Haematobium, occupational exposures to aromatic amines and polycyclic aromatic hydrocarbons (Silverman et al., 2006; Burger et al., 2013), and other possible factors such as diet, environmental pollution, and genetic predisposition; while cigarette smoking is the dominant cause of bladder cancer in the United States and Schistosoma Haematobium the leading cause in most parts of Africa (Parkin, 2006; Burger et al., 2013).

Bladder cancer is highly preventable (Burger et al., 2013). Therefore, the urologists can easily affect the prevention of the cancer through interaction with their patients, primary care physicians and society (Klotz and Brausi, 2015). The Human Development Index (HDI) is a useful classification to compare the cancer globally (Bray et al., 2012; Ghoncheh et al., 2015). As a result, countries are classified in to 4 levels, low, medium, high and very high, by education, life expectancy, and national income. Although communicable diseases and nutrition are common causes of death in countries with a low HDI, Noncommunicable diseases such as cancer have overtaken (Wagner and Brath, 2012). It is predicted that the cancer burden in the world has increased from 12.7 million new cases in 2008 to 22.2 million by 2030 (Bray et al., 2012). It shows an increase in population and changes in the age distribution, the incidence, prevalence, and risk factors. For example, open tobacco use in low- and middle-income countries leads to a major effect on the cancer burden in the coming decades (Giovino et al., 2012). Several studies have shown that survival differences are associated with socioeconomic status of the patients (Coleman et al., 2001; Brookfield et al., 2009; Trinh et al., 2012). People with low socio-economic status experience lower survival due to reduced access to health care, biology of the disease, environmental factors, exposure to tobacco, and health habits (Shackley and Clarke, 2005). Persons with extensive knowledge expose lower to bladder cancer risk factors, including smoking. This leads to lower bladder cancer in them (Yang et al., 2010).

The information on the incidence and mortality of the disease, and its distribution in terms of geographical areas is essential for further studies and better plan for the prevention. There is no study to evaluate the incidence and mortality from the cancer in the world. Considering the relationship between the development and incidence and mortality from the cancer, the aim of the study was to investigate bladder cancer incidence and mortality rates, and their relationship with the HDI in the world.

\section{Materials and Methods}

This study was an ecologic study in the world for assessment the correlation between age-specific incidence and mortality rate (ASR) of Bladder Cancer with Human Development Index (HDI) and its details that include: Life expectancy at birth, Mean years of schooling and Gross national income (GNI) per capita. Data about the age-specific incidence and mortality rate (ASR) for every country for year 2012 get from global cancer project that available in (http://globocan.iarc.fr/Default.aspx) and Human Development Index (HDI) from Human Development Report 2013(Malik, 2013) that include information about HDI and its details for every country in the word for year 2012 .

Method of estimate the age-specific Incidence and mortality rates in global cancer project by international agency for research on cancer

\section{Age-specific incidence rate estimate}

The methods of estimation are country specific and the quality of the estimation depends upon the quality and on the amount of the information available for each country. In theory, there are as many methods as countries, and because of the variety and the complexity of these methods, an overall quality score for the incidence and mortality estimates combined is almost impossible to establish. However an alphanumeric scoring system which independently describes the availability of incidence and mortality data has been established at the country level. The combined score is presented together with the estimates for each country with an aim of providing a broad indication of the robustness of the estimation.

The methods to estimate the sex- and age-specific incidence rates of cancer for a specific country fall into one of the following broad categories, in priority order:

1- Rates projected to 2012 (38 countries)-2- Most recent rates applied to 2012 population (20 countries)3 -Estimated from national mortality by modelling, using incidence mortality ratios derived from recorded data in country-specific cancer registries (13 countries)-4Estimated from national mortality estimates by modelling, using incidence mortality ratios derived from recorded data in local cancer registries in neighboring countries (9 European countries)-5-Estimated from national mortality estimates using modelled survival (32 countries)-6Estimated as the weighted average of the local rates (16 countries)-7- One cancer registry covering part of a country is used as representative of the country profile (11 countries)-8-Age/sex specific rates for "all cancers" were partitioned using data on relative frequency of different cancers (by age and sex) (12 countries)-9- The rates are those of neighboring countries or registries in the same area (33 countries). (Stewart and Wild, 2014; Ferlay et al., 2015) 
Epidemiology, Incidence and Mortality of Bladder Cancer and Relationships with the Development Index in the World

Age-specific mortality rate estimate

Depending of the degree of detail and accuracy of the national mortality data, six methods have been utilized in the following order of priority:

1-Rates projected to 2012 (69 countries)-2- Most recent rates applied to 2012 population (26 countries)-3Estimated as the weighted average of regional rates (1 country)-4- Estimated from national incidence estimates by modelling, using country-specific survival (2 countries)-5-Estimated from national incidence estimates using modelled survival (83 countries)-6-The rates are those of neighboring countries or registries in the same area ( 3 countries)

\section{Human development index (HDI)}

Human Development Index (HDI), a composite measure of indicators along three dimensions: life expectancy, educational attainment and command over the resources needed for a decent living. All groups and regions have seen notable improvement in all HDI components, with faster progress in low and medium HDI countries. On this basis, the world is becoming less unequal. Nevertheless, national averages hide large variations in human experience. Wide disparities remain within countries of both the North and the South, and income inequality within and between many countries has been rising.(Malik, 2013)

Statistical analysis: In this study, we use of correlation bivariate method for assessment the correlation between age-specific incidence and mortality rate (ASR) with Human Development Index (HDI) and its details that include: Life expectancy at birth, Mean years of schooling and Gross national income (GNI) per capita. Statistical significance was assumed if $\mathrm{P}<0.05$. All reported $\mathrm{P}$-values are two-sided. Statistical analyses were performed using SPSS (Version 15.0, SPSS Inc).

\section{Results}

\section{The incidence of bladder cancer}

In 2012,429,793 bladder cancer cases occurred in the world, so that there were 237,778 new cases in countries with very high HDI, 72,293 cases in countries with high HDI, 103,105 cases in countries with moderate HDI, and 16,407 cases in countries with low HDI. The highest number of cancer cases was seen in countries such as the United States with about 68,639 cases, China 55,486, Germany about 28,405, Japan 22,042, and Italy with about 18,284 cases, respectively. Five countries that had the largest number of bladder cancer in men were the United States about 52,099, China about 41,993, Germany 21,656 , Japan 16755, and Italy 14,674 cases, respectively. The highest number in women was seen in 5 countries including the United States about 16,540, China 13,493, Germany 6,749 cases, Japan 5,287, and Italy 3,610 cases, respectively.

\section{The age- standardized incidence rate (ASIR)}

The incidence rate in countries with very high HDI was 9.7 per 100,000 , in countries with a high HDI 5.9 per 100,000 , in countries with medium HDI 2.9 per 100,000 , and in countries with a low HDI 2.2 per 100,000 . Five countries that had the highest age-standardized incidence were

Belgium 17.5 per 100,000 , Lebanon $16.6100,000$, Malta 15.8 per 100,000 , Turkey 15.2 per 100,000 , and Denmark 14.4 per 100,000 , respectively. Five countries with the highest age-standardized incidence rate for men included Belgium 31 per 100,000, Lebanon 29.1 per 100,000 , Turkey 28.7 per 100,000 , Armenia 27.3 per 100,000, and Malta 26.9 per 100,000, respectively. Five countries also had the highest age-standardized incidence rate for women were Hungary 7.4 per 100,000, Denmark 7.0 per 100,000 , Norway 6.4 per 100,000 , Belgium 6.2 per 100,000, and Malta 6.2 per 100,000, respectively. (Figure 1,2)

\section{The number of deaths from cancer}

In 2012, 165,084 bladder death cases occurred in the world, so that there were 71,687 death cases in countries with very high HDI, 30,827 cases in countries with high HDI, 52,995 cases in countries with moderate HDI, and 10,508 cases in countries with low HDI. The highest number of death cases was seen in countries such as China 26,820 cases, the United States 16,468, India 9,523, Japan 7,630 , and Russia 6,843 cases, respectively. Five countries that had the largest number of death in men were China 20,056, the United States 4,669, India 7,664, Russia 5,352, and Japan 5,168 cases, respectively. The highest number in women was seen in 5 countries including China about 6,762, the United States 4,662, Japan 2,462 cases, Germany 1,861, and India 1,859 cases, respectively.

\section{The age-standardized death rate (ASDR)}

The age-standardized death rate in countries with very high HDI was 2.4 per 100,000 , in countries with a high HDI 2.4 per 100,000 , in countries with medium HDI 1.4 per 100,000 , and in countries with a low HDI 1.5 per 100,000 . Five countries that had the highest agestandardized death rate were Turkey 6.6 per 100,000 , Egypt $6.5100,000$, Iraq 6.3 per 100,000 , Lebanon 6.3 per 100,000, and Mali 5.2 per 100,000, respectively. Five countries with the highest age-standardized death rate for men included Turkey 12.8 per 100,000, Iraq 11.4 per 100,000, Armenia 1.3 per 100,000, Egypt 11.1 per 100,000, and Lebanon 11 per 100,000, respectively. Five countries also had the highest age-standardized death rate for women were Mali 4.4 per 100,000, Malawi 4.4 per 100,000, Malta 4.3 per 100,000 , French Polynesia 2.9 per 100,000 , and Iraq 2.9 per 100,000 , respectively. (Figure1,3)

\section{Correlation analysis between the standardized incidence} rate, and HD and its components

The results of correlation analysis showed a positive linear relationship between the standardized incidence rate and HDI ( $\mathrm{r}=0.653, \mathrm{P}<0.001)$. (Figure 4) A positive correlation was observed between HDI components and standardized incidence rate, so that there was a positive correlation between the standardized incidence rate and life expectancy at birth $(\mathrm{r}=0.596, \mathrm{P}<0.001)$, the standardized incidence rate and average years of schooling 
Neda Mahdavifar et al

$(\mathrm{r}=0.578, \mathrm{P}<0.001)$, and the standardized incidence rate and the level of income per person of population $(r=$ $0.487, \mathrm{P}<0.001)$.

Correlation analysis between the standardized mortality rate, and HDI and its components

The results of correlation analysis showed a positive linear relationship between the standardized mortality rate and HDI $(\mathrm{r}=0.308, \mathrm{P}<0.001)$. (Figure 5) A positive correlation was observed between HDI components and standardized mortality rate, so that there was a positive correlation between the standardized mortality rate and life expectancy at birth $(\mathrm{r}=0.292, \mathrm{P}<0.001)$, the standardized mortality rate and average years of schooling ( $\mathrm{r}=0.244$, $\mathrm{P}<0.001)$ and the standardized mortality rate and the level of income per person of population $(\mathrm{r}=0.172, \mathrm{P}<0.001)$.

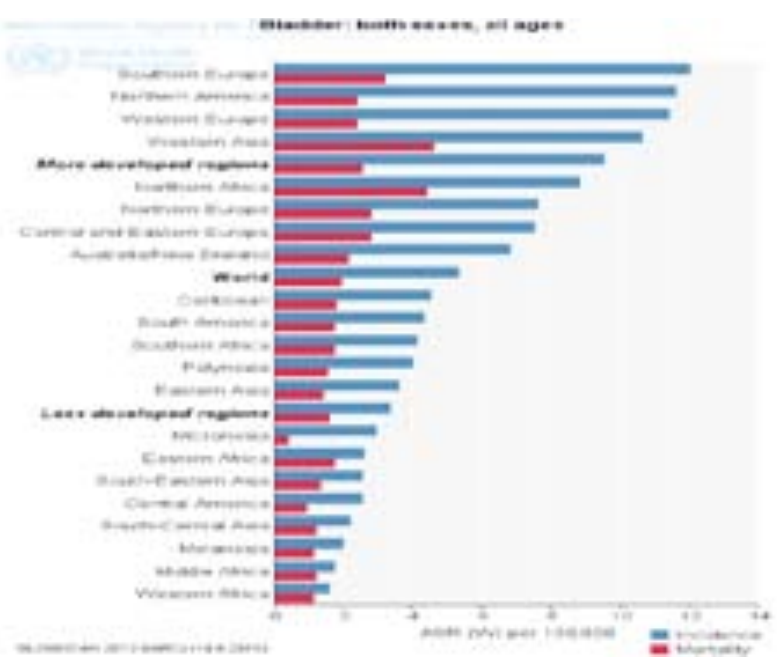

Figure 1.ASDR and ASIR in Different Region of World (Extracted from GLOBOCAN 1012)

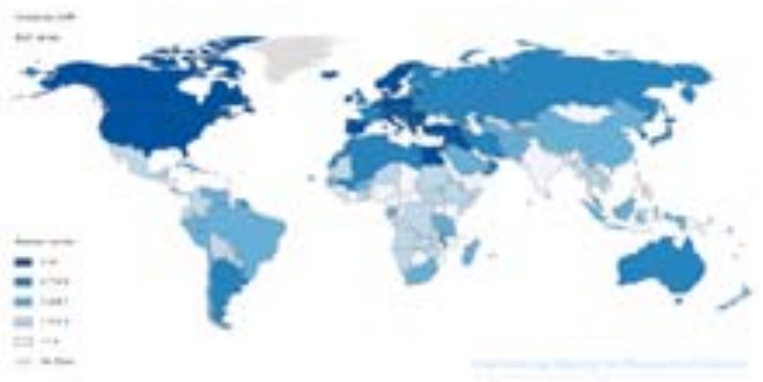

Figure 2. Distribution ASIR in Both Sex in all of World (extracted from GLOBOCAN 1012)

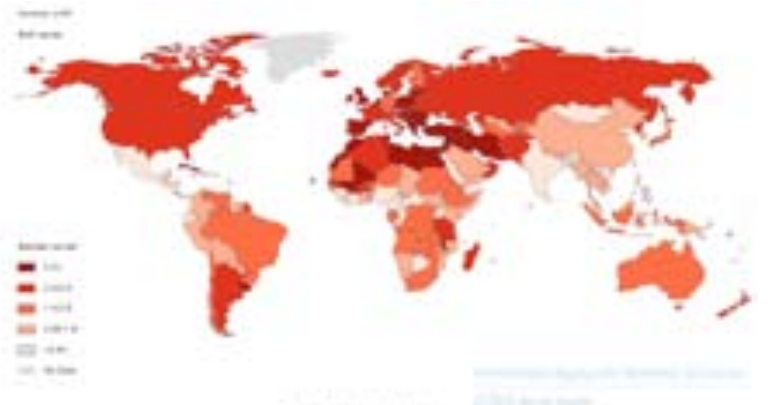

Figure 3. Distribution ASDR in Both Sex in All of World (Extracted from GLOBOCAN

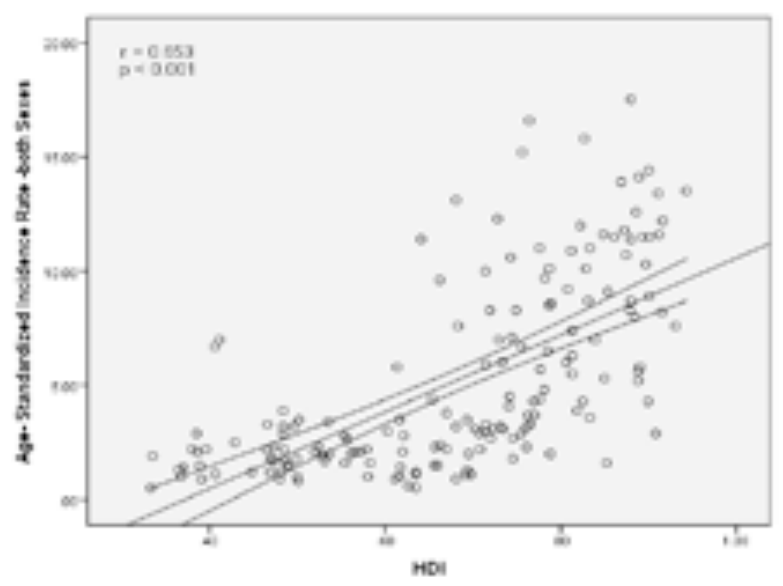

Figure 4. Correlation between HDI and ASIR in Both Sexes in all of World Country

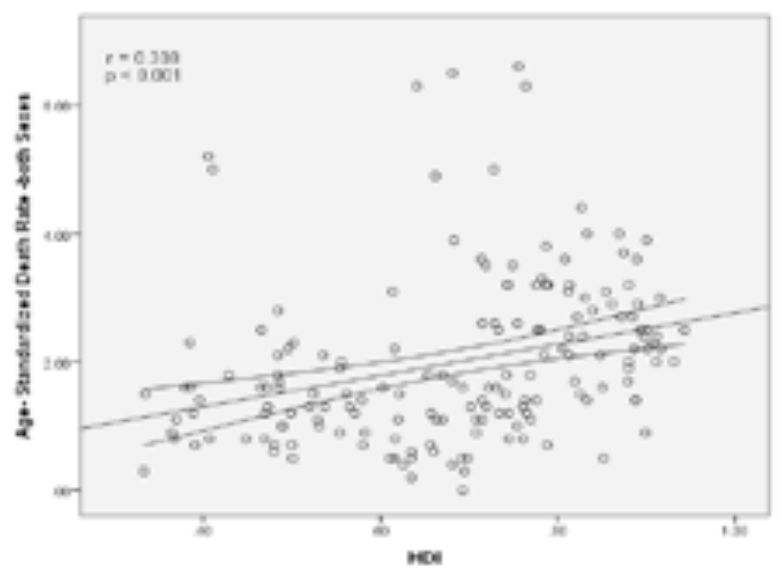

Figure 5: Correlation between HDI and ASDR in both Sexes in All of World

\section{Discussion}

There were more than 900,000 new cases of bladder cancer per year, and approximately 250,000 deaths occur from the cancer worldwide (Klotz and Brausi, 2015). In addition to the human toll, the prevalence of bladder cancer is associated with the economic burden on global health care systems (Measuring the burden is based on the overall cost of patient from diagnosis to death. Bladder cancer is one of the most expensive cancers in terms of treatment (Sievert et al., 2009). Considering the aging population and its impact on the burden of cancer, this cancer is significant and requires special attention (Ploeg et al., 2009).

Our results showed that the highest standardized incidence rate of bladder cancer was related to Belgium, Lebanon, Malta, Turkey, and Denmark, respectively. In this study, a strong correlation was observed between HDI and incidence of bladder cancer. Other studies have also shown that the incidence of bladder cancer was associated with a significant difference in high HDI countries than countries with low HDI, but there was a little difference in terms of mortality rate (Bray et al., 2012).

The highest incidence of bladder cancer was seen in developed countries and parts of Africa, while the highest mortality rate was related to the North African countries of the Middle East. The difference in bladder 
Epidemiology, Incidence and Mortality of Bladder Cancer and Relationships with the Development Index in the World

cancer incidence and mortality in countries around the world is due to differences in known risk factors, coding, detection, diagnosis practices, and access to health care (Chavan et al., 2014). Greater access to health care and better treatment is one of the causes of lower death in countries with higher HDI (Ploeg et al., 2009).

Smoking is the most important risk factors for bladder cancer in most parts of the world (Silverman et al., 2006), so that the attributable risk is $50 \%$ in Europe (Agudo et al., 2012). In a meta-analysis, it has been recently seen that smoking is associated with a threefold increase in the risk of lower urinary tract cancer, particularly bladder (Gandini et al., 2008). About $90 \%$ of cases with bladder cancer result from smoking (Kaufman et al., 2009). The increase in the incidence and mortality in women in some Eastern European countries is because of the prevalence of cigarette smoking(Chavan et al., 2014). Reduced mortality rate in Western countries is due to decreasing the prevalence of tobacco use (Chavan et al., 2014), so that the prevalence of smoking decreased in American adults in 1965 from about $45 \%$ to about $19 \%$ in 2010 (Jemal et al., 2008). As well as, a significant reduction has also reported from Canada and Australia (Gilmore, 2002; July 24,2012 ). In developed and Western countries, few studies showed that the reduction in the incidence of bladder cancer has been attributed to decreasing of occupational exposures (Pelucchi et al., 2006; Ferlay et al., 2008; Cirla et al., 2011). A relatively high incidence of bladder cancer in North Africa has been largely attributed to the parasite Schistosoma Haematobium. Egypt is a good example in this regard so that the control of S. Haematobium declined cases of bladder cancer (Salem and Mahfouz, 2012). Reduced mortality in these countries is due to controlling S. Haematobium and improving sanitation and hygiene (Chavan et al., 2014).

In our study, a significant positive relationship was observed between life expectancy as one of the components of the HDI and the standardized incidence and mortality rates. In other studies, with increased average life expectancy, the incidence of bladder cancer increased in the elderly (Yuge et al., 2011). Life expectancy is more in women than in men with similar age and race. For example, in 2006, life expectancy for a 70 year old white men with bladder cancer was 13.6 , but in a white women 70 years 15.9 (March 2011). It can be concluded that bladder cancer is related to elderly. Considering aging populations, the incidence of the cancer will increase. Hence, it is necessary to pay more attention and studies on the etiology.

One of the components of HDI is access to knowledge, which is specified by the average years of schooling. In our study, a significant positive correlation was observed between the education level and standardized incidence and mortality rates. Other studies also found a significant positive correlation between level of education and bladder cancer(Hussain et al., 2008) . Education levels may be related to behavior, health conditions, access to knowledge, and resources that directly or indirectly affects the cancer (Spiegel et al., 1989; Kogevinas M, 1997; Spiegel, 2002; Spiegel and Giese-Davis, 2003; Lehto et al., 2006; van Vliet et al., 2006; Griggs et al., 2007).
Another component of HDI is the income level, which is determined by the Gross National Product (GDP). In our study, there was a significant positive relationship between the income level, and the standardized incidence and mortality rates. A strong correlation between socioeconomic status and survival of patients with bladder cancer has been observed in other studies. In other words, the increase in premature death was seen in patients with low socioeconomic status than those with higher socioeconomic status (Siddiqui et al., 2015). The patients with low socio-economic status experience lower survival due to reduced access to health care, biology of the disease, environmental factors, exposure to tobacco, and health habits (Shackley and Clarke, 2005). Thus, reducing the incidence and mortality from the cancer in all countries is possible using interventions such as tobacco control, improved sanitation and hygiene to reduce infection with S. Haematobium, improving safety of workplaces, and decreasing occupational exposures (Chavan et al., 2014).

In conclusion, The incidence of bladder cancer in developed countries and parts of Africa was higher, while the highest mortality rate was observed in the countries of North Africa and the Middle East there was statistically a significant positive correlation between the standardized incidence rate of bladder cancer, and the HDI, and its components, including life expectancy at birth, the average level of education, and the income level per person. A significant positive correlation was observed between standardized mortality rate, and the HDI and its components. The program for better treatment in developing countries to reduce mortality from the cancer and studies on the etiology of incidence and mortality are essential.

\section{References}

Agudo A, Bonet C, Travier N, et al (2012). Impact of cigarette smoking on cancer risk in the European prospective investigation into cancer and nutrition study. J Clin Oncol, 30, 4550-7.

Brausi M, Witjes JA, Lamm D, et al (2011). A review of current guidelines and best practice recommendations for the management of nonmuscle invasive bladder cancer by the International Bladder Cancer Group. J Urol, 186, 2158-67.

Bray F, Jemal A, Grey N, et al (2012). Global cancer transitions according to the Human Development Index (2008-2030): a population-based study. The Lancet Oncol, 13, 790-801.

Brookfield KF, Cheung MC, Gomez C, et al (2009). Survival disparities among African American women with invasive bladder cancer in Florida. Cancer, 115, 4196-209.

Burger M, Catto JW, Dalbagni G, et al (2013). Epidemiology and risk factors of urothelial bladder cancer. European urology, 63, 234-41.

Chavan S, Bray F, Lortet-Tieulent J, et al (2014). International variations in bladder cancer incidence and mortality. European Urology, 66, 59-73.

Cirla PE, Martinotti I, Foa V (2011). [Occupational exposure to chemical carcinogens between estimates and reality: an Italian multicentric study in Lombardy]. G Ital Med Lav Ergon, 33, 85-7.

Coleman MP, Babb P, Sloggett A, et al (2001). Socioeconomic inequalities in cancer survival in England and Wales. Cancer, 91, 208-16. 
Neda Mahdavifar et al

Colombel M, Soloway M, Akaza H, et al (2008). Epidemiology, staging, grading, and risk stratification of bladder cancer. European Urology Ssupplements, 7, 618-26.

Ferlay J, Randi G, Bosetti C, et al (2008). Declining mortality from bladder cancer in Europe. BJU international, 101, 11-9.

Ferlay J, Soerjomataram I, Dikshit R, et al (2015). Cancer incidence and mortality worldwide: sources, methods and major patterns in GLOBOCAN 2012. International Journal of Cancer, 136, 359-86.

Gandini S, Botteri E, Iodice S, et al (2008). Tobacco smoking and cancer: a meta-analysis. Int J Cancer, 122, 155-64.

Ghoncheh M, Mohammadian-Hafshejani A, Salehiniya H (2015). Incidence and Mortality of Breast Cancer and their Relationship to Development in Asia. Asian Pac J Cancer Prev, 16, 6081-7.

Gilmore J (2002). Report on smoking prevalence in Canada. Statistics Canada, Ottawa.

Giovino GA, Mirza SA, Samet JM, et al (2012). Tobacco use in 3 billion individuals from 16 countries: an analysis of nationally representative cross-sectional household surveys. The Lancet, 380, 668-79.

Griggs JJ, Culakova E, Sorbero ME, et al (2007). Effect of patient socioeconomic status and body mass index on the quality of breast cancer adjuvant chemotherapy. J Clin Oncol, 25, 277-84.

Hussain SK, Lenner P, Sundquist J, et al (2008). Influence of education level on cancer survival in Sweden. Ann Oncol, 19, $156-62$.

Jemal A, Thun MJ, Ries LA, et al (2008). Annual report to the nation on the status of cancer, 1975-2005, featuring trends in lung cancer, tobacco use, and tobacco control. J National Cancer Institute, 100, 1672-94.

Kaufman DS, Shipley WU, Feldman AS (2009). Bladder cancer. Lancet, 374, 239-49.

Keyghobadi N, Rafiemanesh H, Mohammadian-Hafshejani A, et al (2015). Epidemiology and trend of cancers in the province of Kerman: southeast of Iran. Asian Pac J Cancer Prev, 16, 1409-13.

Klotz L, Brausi MA (2015). World urologic oncology federation bladder cancer prevention program: a global initiative. urologic oncology: seminars and original investigations. Elsevier, 25-9.

Kogevinas M PN, Susser M, et al (1997). Social Inequalities and Cancer. Lyon, France. IARC Scientific Publications.

Lehto US, Ojanen M, Dyba T, et al (2006). Baseline psychosocial predictors of survival in localised breast cancer. Br J Cancer, 94, 1245-52.

Malats N, Real FX (2015). Epidemiology of Bladder Cancer. Hematology/oncology clinics of North America, 29, 177-89.

Malik K (2013). Human development report 2013. The rise of the South: Human progress in a diverse world. The Rise of the South: Human Progress in a Diverse World (March 15, 2013). UNDP-HDRO Human Development Reports.

Parkin DM (2006). The global health burden of infection associated cancers in the year 2002. Intern J Cancer, 118, 3030-44.

Pelucchi C, Bosetti C, Negri E, et al (2006). Mechanisms of disease: The epidemiology of bladder cancer. Nat Clin Pract Urol, 3, 327-40.

Ploeg M, Aben KK, Kiemeney LA (2009). The present and future burden of urinary bladder cancer in the world. World J Urol, 27, 289-93.

Razi S, Rafiemanesh H, Ghoncheh M, et al (2015). Changing Trends of Types of Skin Cancer in Iran. Asian Pac J Cancer Prev, 16, 4955-8.

Salem HK, Mahfouz S (2012). Changing patterns (age, incidence, and pathologic types) of schistosoma-associated bladder cancer in Egypt in the past decade. Urology, 79, 379-83.

Shackley DC, Clarke NW (2005). Impact of socioeconomic status on bladder cancer outcome. Current Opinion in Urol, 15, 328-31.

Siddiqui MM, Heney NM, McDougal WS, et al (2015). Disparities in overall and urothelial carcinoma specific mortality associated with healthcare insurance status. Bladder, 2, 10.

Sievert KD, Amend B, Nagele U, et al (2009). Economic aspects of bladder cancer: what are the benefits and costs? World $J$ Urol, 27, 295-300.

Silverman D, Devesa S, Moore L, et al (2006). Cancer epidemiology and prevention. Bladder Cancer, 2, 1156-79.

Spiegel D (2002). Effects of psychotherapy on cancer survival. Nat Rev Cancer, 2, 383-9.

Spiegel D, Bloom JR, Kraemer HC, et al (1989). Effect of psychosocial treatment on survival of patients with metastatic breast cancer. Lancet, 2, 888-91.

Spiegel D, Giese-Davis J (2003). Depression and cancer: mechanisms and disease progression. Biol Psychiatry, 54, 269-82.

Trinh QD, Schmitges J, Sun M, et al (2012). Morbidity and mortality of radical prostatectomy differs by insurance status. Cancer, 118, 1803-10.

van Vliet EP, Eijkemans MJ, Steyerberg EW, et al (2006). The role of socio-economic status in the decision making on diagnosis and treatment of oesophageal cancer in The Netherlands. Br J Cancer, 95, 1180-5.

Wagner K-H, Brath H (2012). A global view on the development of non communicable diseases. Preventive medicine, 54, $38-41$.

Yang Y, Wang JJ, Wang CX, et al (2010). Awareness of tobaccorelated health hazards among adults in China. Biomed Environ Sci, 23, 437-44.

Yuge K, Kikuchi E, Matsumoto K, et al (2011). Could patient age influence tumor recurrence rate in non-muscle-invasive bladder cancer patients treated with BCG immunotherapy? Jpn J Clin Oncol, 41, 565-70. 